\title{
Chronology of 1940s American Culture
}




\begin{tabular}{|c|c|c|c|c|}
\hline Date & Events & Criticism & Literature & Performance \\
\hline 1940 & $\begin{array}{l}1940 \text { Olympics } \\
\text { cancelled. } \\
\text { Nylons sold as } \\
\text { substitute for silk } \\
\text { stockings. } \\
\text { Germans enter Paris } \\
\text { Battle of Britain } \\
\text { (July-October). } \\
\text { Forty-hour work } \\
\text { week implemented, } \\
\text { under the Fair Labor } \\
\text { Standards Act of } \\
\text { 1938. } \\
\text { Roosevelt wins third } \\
\text { term in office, } \\
\text { defeating Wendell } \\
\text { Willkie. } \\
\text { Roosevelt in radio } \\
\text { discussion proclaims } \\
\text { US 'arsenal of } \\
\text { democracy'. }\end{array}$ & $\begin{array}{l}\text { Lewis Mumford, } \\
\text { Faith for Living } \\
\text { W. E. B. Du Bois, } \\
\text { Dusk of Dawn } \\
\text { Alaine Locke, The } \\
\text { Negro in Art }\end{array}$ & $\begin{array}{l}\text { Richard Wright, } \\
\text { Native Son } \\
\text { Ernest Hemingway, } \\
\text { For Whom the Bell } \\
\text { Tolls } \\
\text { Raymond Chandler, } \\
\text { Farewell, My Lovely } \\
\text { Walter Clark, The } \\
\text { Ox-Bow Incident } \\
\text { Carson McCullers, } \\
\text { The Heart is a } \\
\text { Lonely Hunter } \\
\text { John Steinbeck wins } \\
\text { Pulitzer Prize for } \\
\text { Grapes of Wrath } \\
\text { F. Scott Fitzgerald } \\
\text { dies of a heart attack } \\
\text { at forty-four }\end{array}$ & $\begin{array}{l}\text { Richard Rodgers } \\
\text { and Lorenz Hart, } \\
\text { Higher and Higher } \\
\text { (musical) } \\
\text { Vernon Duke and } \\
\text { John Latouche, } \\
\text { Cabin in the Sky } \\
\text { (musical) } \\
\text { Cole Porter, Panama } \\
\text { Hattie (musical) } \\
\text { Richard Rodgers } \\
\text { and Lorenz Hart, } \\
\text { Pal Joey (musical) }\end{array}$ \\
\hline
\end{tabular}




\begin{tabular}{|c|c|c|c|}
\hline Film & Radio & Music & Art \\
\hline $\begin{array}{l}1939 \text { Oscars awarded - } \\
\text { Gone with the Wind, } \\
\text { wins eight awards, } \\
\text { including Best Picture, } \\
\text { Best Actress (Vivian } \\
\text { Leigh) and Best } \\
\text { Supporting Actress } \\
\text { (Hattie McDaniel) } \\
\text { The Grapes of Wrath } \\
\text { (John Ford) } \\
\text { Foreign Correspondent } \\
\text { and Rebecca (Alfred } \\
\text { Hitchcock) } \\
\text { The Philadelphia Story } \\
\text { (George Cukor) } \\
\text { His Girl Friday } \\
\text { (Howard Hawks) } \\
\text { Charles Chaplin's The } \\
\text { Great Dictator opens } \\
\text { at two cinemas in New } \\
\text { York; Chaplin and his } \\
\text { wife, co-star Paulette } \\
\text { Goddard, make } \\
\text { appearances at both } \\
\text { Pinocchio and Fantasia } \\
\text { (Walt Disney) } \\
\text { Tom and Jerry (Hanna- } \\
\text { Barbera) } \\
\text { Weekly cinema } \\
\text { attendance: eighty } \\
\text { million }\end{array}$ & $\begin{array}{l}\text { The Adventures of } \\
\text { Superman (1940-51) } \\
\text { Truth or Consequences } \\
\text { (1940-57) } \\
\text { Take It or Leave It, } \\
\text { later The } \$ 64,000 \\
\text { Question }(1940-52) \\
\text { The Bell Telephone } \\
\text { Hour, concert series } \\
\text { (1940-58) } \\
\text { Edward R. Murrow's } \\
\text { London Broadcasts }\end{array}$ & $\begin{array}{l}\text { Glenn Miller } \\
\text { Orchestra's 'In The } \\
\text { Mood' number one } \\
\text { Woody Guthrie writes } \\
\text { 'This Land Is Your } \\
\text { Land' } \\
\text { Artie Shaw Orchestra } \\
\text { records Hoagy } \\
\text { Carmichael's 'Stardust' } \\
\text { for RCA Victor } \\
\text { Frank Sinatra joins } \\
\text { Tommy Dorsey's } \\
\text { Orchestra } \\
\text { Thelonius Monk } \\
\text { begins performing }\end{array}$ & $\begin{array}{l}\text { American Negro } \\
\text { Exhibition } \\
\text { Edward Hopper, Gas } \\
\text { Brenda Starr, first } \\
\text { comic strip by a } \\
\text { woman } \\
\text { In Batman (comic } \\
\text { strip) mobsters do } \\
\text { away with a circus } \\
\text { family known as the } \\
\text { Flying Graysons, } \\
\text { leaving their son Dick } \\
\text { (Robin) an orphan }\end{array}$ \\
\hline
\end{tabular}




\begin{tabular}{|c|c|c|c|c|}
\hline Date & Events & Criticism & Literature & Performance \\
\hline 1941 & $\begin{array}{l}\text { Tuskegee Airmen (all- } \\
\text { black flying unit) } \\
\text { commissioned; will } \\
\text { fly their first mission } \\
\text { in June } 1943 \text {. } \\
\text { FDR delivers 'Four } \\
\text { Freedoms' speech in } \\
\text { State of the Union } \\
\text { address. } \\
\text { Congress approves } \\
\text { the Lend-Lease Act. } \\
\text { Cheerios introduced. } \\
\text { FDR signs Executive } \\
\text { Order } 8803 \text {, barring } \\
\text { racial discrimination } \\
\text { in the war-industry } \\
\text { workplace. } \\
\text { Mount Rushmore } \\
\text { completed. } \\
\text { Japanese attack Pearl } \\
\text { Harbor (7 December, } \\
7.50 \text { a.m.). } \\
\text { Alexander Fleming } \\
\text { discovers penicillin. } \\
\text { Manhattan Project } \\
\text { begins. }\end{array}$ & $\begin{array}{l}\text { Henry R. Luce, 'The } \\
\text { American Century' } \\
\text { Erich Fromm, } \\
\text { Escape from } \\
\text { Freedom } \\
\text { John Crowe } \\
\text { Ransom, The New } \\
\text { Criticism }\end{array}$ & $\begin{array}{l}\text { James Agee, Let Us } \\
\text { Now Praise Famous } \\
\text { Men (non-fiction) } \\
\text { William Shirer, } \\
\text { Berlin Diary } \\
\text { Eudora Welty, A } \\
\text { Curtain of Green } \\
\text { (short fiction) } \\
\text { F. Scott Fitzgerald, } \\
\text { The Last Tycoon } \\
\text { James M. Cain, } \\
\text { Mildred Pierce } \\
\text { H. A. Rey and } \\
\text { Margaret Rey, } \\
\text { Curious George } \\
\text { (children's story) }\end{array}$ & $\begin{array}{l}\text { Lillian Hellman, } \\
\text { Watch on the Rhine } \\
\text { Joseph Kesselring, } \\
\text { Arsenic and Old } \\
\text { Lace } \\
\text { Kurt Weill, Ira } \\
\text { Gershwin, Moss } \\
\text { Hart, Lady in the } \\
\text { Dark } \\
\text { Eugene O’Neill } \\
\text { completes Long } \\
\text { Day's Journey into } \\
\text { Night (first } \\
\text { performed 1956) }\end{array}$ \\
\hline
\end{tabular}




\begin{tabular}{|c|c|c|c|}
\hline Film & Radio & Music & Art \\
\hline $\begin{array}{l}1940 \text { Oscars awarded - } \\
\text { Best Picture: Alfred } \\
\text { Hitchcock's Rebecca; } \\
\text { Best Director: John } \\
\text { Ford (The Grapes of } \\
\text { Wrath) } \\
\text { Citizen Kane (Orson } \\
\text { Welles) } \\
\text { The Maltese Falcon } \\
\text { (John Huston) } \\
\text { How Green was My } \\
\text { Valley (John Ford) } \\
\text { The Lady Eve (Preston } \\
\text { Sturges) } \\
\text { Meet John Doe (Frank } \\
\text { Capra) } \\
\text { The Little Foxes } \\
\text { (William Wyler) } \\
\text { Sergeant York } \\
\text { (Howard Hawks) }\end{array}$ & $\begin{array}{l}\text { Inner Sanctum, } \\
\text { mystery series } \\
(1941-52) \\
\text { Duffy's Tavern, } \\
\text { situation comedy } \\
\text { (1941-52) } \\
\text { The Great Gildersleeve } \\
\text { (1941-57) } \\
\text { A Date with Judy } \\
\text { (1941-49) } \\
\text { The Life of Riley } \\
\text { (1941-51) } \\
\text { Dave Garroway hosts } \\
\text { his first jazz } \\
\text { programme for troops } \\
\text { serving in US Navy } \\
\text { Bob Hope's first } \\
\text { performance for troops } \\
\text { takes place as a } \\
\text { Pepsodent Show } \\
\text { broadcast live from } \\
\text { Riverside California's } \\
\text { March Field } \\
\text { Fireside Chat, FDR } \\
\text { discusses declaration of } \\
\text { war on Japan (9 } \\
\text { December ) }\end{array}$ & $\begin{array}{l}\text { Ethno-musicologist } \\
\text { Alan Lomax meets } \\
\text { blues artists Muddy } \\
\text { Waters and Son House } \\
\text { Duke Ellington, 'Take } \\
\text { the A Train' } \\
\text { The Andrew Sisters, } \\
\text { 'Boogie Woogie Bugle } \\
\text { Boy' } \\
\text { Benny Goodman } \\
\text { integrates his big band }\end{array}$ & $\begin{array}{l}\text { National Gallery of } \\
\text { Art opens in } \\
\text { Washington, DC } \\
\text { Adolph Gottlieb } \\
\text { begins Pictograph } \\
\text { series (1941-50) } \\
\text { Robert Motherwell } \\
\text { moves to Greenwich } \\
\text { Village and decides to } \\
\text { paint full-time } \\
\text { Wonder Woman (comic } \\
\text { book) is first published } \\
\text { Captain America } \\
\text { (comic book) is first } \\
\text { published }\end{array}$ \\
\hline
\end{tabular}




\begin{tabular}{|c|c|c|c|c|}
\hline Date & Events & Criticism & Literature & Performance \\
\hline 1942 & 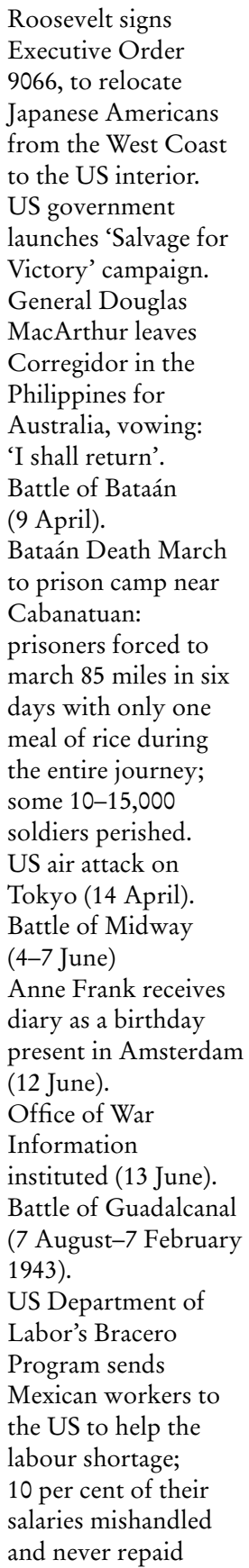 & $\begin{array}{l}\text { Earl Brown, } \\
\text { 'American Negroes } \\
\text { and the War' } \\
\text { Philip Wylie, } A \\
\text { Generation of Vipers }\end{array}$ & $\begin{array}{l}\text { Marion Hargrove, } \\
\text { See Here, Private } \\
\text { Hargrove (comic } \\
\text { non-fiction) } \\
\text { John Steinbeck, The } \\
\text { Moon is Down } \\
\text { (novel) } \\
\text { The New York } \\
\text { Times inaugurates its } \\
\text { Bestseller List } \\
\text { Marjorie Kinnan } \\
\text { Rawlings, Cross } \\
\text { Creek }\end{array}$ & $\begin{array}{l}\text { Thornton Wilder, } \\
\text { The Skin of Our } \\
\text { Teeth (comedy) } \\
\text { Richard Rodgers } \\
\text { and Lorenz Hart, } \\
\text { By Jupiter } \\
\text { Cole Porter, Du } \\
\text { Barry was a Lady } \\
\text { Irving Berlin, This is } \\
\text { the Army } \\
\text { Eugene O'Neill, } \\
\text { A Touch of the Poet } \\
\text { (first performed } \\
\text { 1958) }\end{array}$ \\
\hline
\end{tabular}




\begin{tabular}{|c|c|c|c|}
\hline Film & Radio & Music & Art \\
\hline $\begin{array}{l}\text { Actress Carol } \\
\text { Lombard (wife of } \\
\text { Clark Gable) killed } \\
\text { with twenty others in } \\
\text { an aeroplane crash on a } \\
\text { war bond-selling tour } \\
1941 \text { Oscars awarded - } \\
\text { Best Picture and Best } \\
\text { Director: How Green } \\
\text { Was My Valley (beating } \\
\text { Citizen Kane) } \\
\text { The Pride of the } \\
\text { Yankees (Sam Wood) } \\
\text { Casablanca (Mchael } \\
\text { Curtiz) } \\
\text { Mrs. Miniver (William } \\
\text { Wyler) } \\
\text { The Road to Morocco } \\
\text { stars Bing Crosby, Bob } \\
\text { Hope and Dorothy } \\
\text { Lamour }\end{array}$ & $\begin{array}{l}\text { Voice of America } \\
\text { begins broadcasting } \\
\text { Suspense (1942-62) } \\
\text { The Cisco Kid } \\
\text { (1942-56) } \\
\text { It Pays to Be Ignorant } \\
\text { (1942-51) } \\
\text { Mr. and Mrs. North } \\
\text { (1942-54) } \\
\text { The March of Time } \\
\text { switches from } \\
\text { dramatisations of } \\
\text { current events to actual } \\
\text { news reporting }\end{array}$ & $\begin{array}{l}\text { Count Basie Orchestra, } \\
\text { 'One O'Clock Jump' - } \\
\text { Basie Orchestra the } \\
\text { first African American } \\
\text { swing band to play at } \\
\text { New York's Hotel } \\
\text { Lincoln } \\
\text { Glenn Miller } \\
\text { Orchestra, '(I've Got a } \\
\text { Gal in) Kalamazoo' } \\
\text { Benny Goodman, } \\
\text { 'Jersey Bounce' } \\
\text { American Federation } \\
\text { of Musicians' strike } \\
\text { (1942-43) } \\
\text { Aaron } \\
\text { Copeland/Agnes de } \\
\text { Mille, Rodeo, New } \\
\text { York Metropolitan } \\
\text { Opera House } \\
\text { Aaron Copeland, } \\
\text { Fanfare for the } \\
\text { Common Man }\end{array}$ & $\begin{array}{l}\text { Edward Hopper, } \\
\text { Nightharwks } \\
\text { Norman Rockwell, } \\
\text { Four Freedoms } \\
\text { Peggy Guggenheim } \\
\text { opens Art of This } \\
\text { Century Gallery } \\
\text { (1942-47) } \\
\text { First Papers of } \\
\text { Surrealism exhibition } \\
\text { at Whitelaw Reid } \\
\text { Mansion in New York } \\
\text { VVV, a journal } \\
\text { devoted to surrealism, } \\
\text { opens (1942-44) } \\
\text { Archie (comic book) } \\
\text { begins }\end{array}$ \\
\hline
\end{tabular}




\begin{tabular}{|c|c|c|c|c|}
\hline Date & Events & Criticism & Literature & Performance \\
\hline 1943 & $\begin{array}{l}\text { Frankfurters replaced } \\
\text { by Victory Sausage, a } \\
\text { mixture of meat and } \\
\text { soya meal. } \\
\text { To cut down on the } \\
\text { need for metal } \\
\text { replacement parts at } \\
\text { bakeries, government } \\
\text { bans sliced bread. } \\
\text { Battle of Anzio, Italy } \\
\text { (22 January). } \\
\text { US begins food- } \\
\text { rationing programme. } \\
\text { Riot, Mobile, } \\
\text { Alabama shipyard } \\
\text { over upgrading twelve } \\
\text { black workers } \\
\text { (25 May). } \\
\text { Race riot, Detroit, } \\
\text { resulting in thirty- } \\
\text { four deaths and six } \\
\text { hundred injured. } \\
\text { Allied invasion of } \\
\text { Italy, Patton crosses } \\
\text { Sicily (July-August) } \\
\text { Race riot, Harlem, } \\
\text { resulting in several } \\
\text { deaths (1 August). } \\
\text { Patton slaps a private, } \\
\text { a shell-shocked } \\
\text { soldier in Sicily, } \\
\text { accusing him of } \\
\text { cowardice. } \\
\text { Italian surrender } \\
\text { (8 September). } \\
\text { Battle of Salerno, } \\
\text { Italy, against Germans } \\
\text { (9-14 September). }\end{array}$ & $\begin{array}{l}\text { Wendell Willkie, } \\
\text { One World } \\
\text { Cleanth Brooks and } \\
\text { Robert Penn } \\
\text { Warren, } \\
\text { Understanding } \\
\text { Fiction } \\
\text { James A. Porter's } \\
\text { Modern Negro Art }\end{array}$ & $\begin{array}{l}\text { Isaac Bashevis Singer } \\
\text { becomes naturalised } \\
\text { US citizen } \\
\text { Ayn Rand, The } \\
\text { Fountainhead } \\
\text { William Saroyan, } \\
\text { The Human } \\
\text { Comedy }\end{array}$ & $\begin{array}{l}\text { Richard Rodgers } \\
\text { and Oscar } \\
\text { Hammerstein II, } \\
\text { Oklahoma! } \\
\text { (musical) } \\
\text { Oscar Hammerstein, } \\
\text { Carmen Jones } \\
\text { (musical) } \\
\text { Kurt Weill and } \\
\text { Ogden Nash, One } \\
\text { Touch of Venus } \\
\text { (comedy) } \\
\text { George Gershwin, } \\
\text { Porgy and Bess } \\
\text { (musical) } \\
\text { Thornton Wilder, } \\
\text { The Skin of Our } \\
\text { Teeth }\end{array}$ \\
\hline
\end{tabular}




\begin{tabular}{|c|c|c|c|}
\hline Film & Radio & Music & Art \\
\hline $\begin{array}{l}1942 \text { Oscars awarded - } \\
\text { Mrs. Miniver wins Best } \\
\text { Picture, Best Director } \\
\text { (William Wyler), and } \\
\text { Best Actress (Greer } \\
\text { Garson) } \\
\text { Cabin in the Sky } \\
\text { (Vincente Minnelli) } \\
\text { Heaven Can Wait } \\
\text { (Ernst Lubitsch) } \\
\text { Shadow of a Doubt } \\
\text { (Alfred Hitchcock) }\end{array}$ & $\begin{array}{l}\text { The Adventures of } \\
\text { Nero Wolfe (1943-44) } \\
\text { The Billie Burke Show } \\
\text { (1943-46) } \\
\text { The Judy Canova } \\
\text { Show (1943-55) } \\
\text { Edward R. Murrow's } \\
\text { 'Orchestrated Hell' } \\
\text { broadcast }\end{array}$ & $\begin{array}{l}\text { Duke Ellington, Black, } \\
\text { Brown and Beige, (jazz } \\
\text { composition) Carnegie } \\
\text { Hall, for Russian War } \\
\text { Relief } \\
\text { Band-leader/trumpet- } \\
\text { player Harry James } \\
\text { weds Betty Grable } \\
\text { Frank Sinatra on Your } \\
\text { Hit Parade (radio } \\
\text { programme); his } \\
\text { 1 January performance } \\
\text { at the Paramount } \\
\text { Theatre caused } \\
\text { hysterical bobby- } \\
\text { soxers to tie up New } \\
\text { York traffic for hours } \\
\text { Miles Davis's career } \\
\text { begins }\end{array}$ & $\begin{array}{l}\text { Norman Rockwell's } \\
\text { Rosie the Riveter on } \\
\text { the cover of The } \\
\text { Saturday Evening Post } \\
\text { Jacob Lawrence, } \\
\text { Ironers } \\
\text { Paul Cadmus, } \\
\text { The Shower } \\
\text { In Washington DC, the } \\
\text { Jefferson Memorial and } \\
\text { Pentagon are } \\
\text { completed } \\
\text { (architecture) }\end{array}$ \\
\hline
\end{tabular}




\begin{tabular}{|c|c|c|c|c|}
\hline Date & Events & Criticism & Literature & Performance \\
\hline 1944 & $\begin{array}{l}\text { Congress passes } \\
\text { Servicemen's } \\
\text { Readjustment Act } \\
\text { (aka the GI Bill of } \\
\text { Rights). } \\
\text { D-Day - one million } \\
\text { Allied troops land at } \\
\text { the beaches of } \\
\text { Normandy, France } \\
\text { (6 June). } \\
\text { Liberation of Paris } \\
\text { (29 August). } \\
\text { African American } \\
\text { petty officer Robert } \\
\text { Jones, USS Intrepid, } \\
\text { shoots down } \\
\text { kamikaze pilot, } \\
\text { awarded the Medal of } \\
\text { Honor in 1997 } \\
\text { (29 October). } \\
\text { Battle of the Bulge (16 } \\
\text { December-16 January } \\
\text { 1945). } \\
\text { Eisenhower invites } \\
\text { African Americans } \\
\text { into combat units; } \\
\text { 2,221 signed up, } \\
\text { giving up military } \\
\text { rank to replace white } \\
\text { casualties. } \\
\text { Lieutenant Jackie } \\
\text { Robinson, US Army, } \\
\text { refuses to give his seat } \\
\text { to a white man, court- } \\
\text { marshalled and } \\
\text { acquitted. } \\
\text { Roosevelt wins fourth } \\
\text { term, defeats Thomas } \\
\text { E. Dewey. } \\
\text { US Army announces } \\
\text { polyethylene } \\
\text { invented. }\end{array}$ & $\begin{array}{l}\text { Gunnar Myrdal, An } \\
\text { American Dilemma } \\
\text { (non-fiction) } \\
\text { Reinhold Niebuhr, } \\
\text { The Children of } \\
\text { Light and the } \\
\text { Children of } \\
\text { Darkness } \\
\text { Lewis Mumford, } \\
\text { Condition of Man }\end{array}$ & $\begin{array}{l}\text { Saul Bellow, } \\
\text { Dangling Man } \\
\text { Lillian Smith, } \\
\text { Strange Fruit } \\
\text { Betty Smith, A Tree } \\
\text { Grows in Brooklyn }\end{array}$ & $\begin{array}{l}\text { Lillian Hellman The } \\
\text { Searching Wind } \\
\text { (drama) } \\
\text { Harold Arlen and } \\
\text { E. Y. Harburg, } \\
\text { Bloomer Girl } \\
\text { Cole Porter, } \\
\text { Mexican Hayride } \\
\text { Leonard Bernstein, } \\
\text { Betty Comden and } \\
\text { Adolph Green, On } \\
\text { the Town } \\
\text { American Negro } \\
\text { Theater's Anna } \\
\text { Lucasta (musical) } \\
\text { opens on Broadway }\end{array}$ \\
\hline
\end{tabular}




\begin{tabular}{|c|c|c|c|}
\hline Film & Radio & Music & Art \\
\hline $\begin{array}{l}1943 \text { Oscars awarded - } \\
\text { Casablanca wins Best } \\
\text { Picture and Best } \\
\text { Director (Michael } \\
\text { Curtiz) } \\
\text { Double Indemnity } \\
\text { (Billy Wilder) } \\
\text { Gaslight (George } \\
\text { Cukor) } \\
\text { Arsenic and Old Lace } \\
\text { (Frank Capra) } \\
\text { Hail the Conquering } \\
\text { Hero (Preston Sturges) } \\
\text { Laura (Otto } \\
\text { Preminger) } \\
\text { Lifeboat (Alfred } \\
\text { Hitchcock) } \\
\text { Since You Went Away } \\
\text { (John Cromwell) } \\
\text { To Have and Have } \\
\text { Not (Howard Hawks) }\end{array}$ & $\begin{array}{l}\text { The Adventures of } \\
\text { Ozzie and Harriet } \\
\text { (1944-54) } \\
\text { The black maid } \\
\text { character Beulah } \\
\text { (played by white male } \\
\text { actor Marlin Hunt) } \\
\text { first appears on Fibber } \\
\text { McGee and Molly } \\
\text { (1935-59) }\end{array}$ & $\begin{array}{l}\text { Leonard Bernstein, } \\
\text { Jeremiah (classical } \\
\text { composition) } \\
\text { Leadbelly, 'Goodnight } \\
\text { Irene' } \\
\text { Aaron Copeland and } \\
\text { Martha Graham, } \\
\text { Appalachian Spring } \\
\text { Big band leader Glenn } \\
\text { Mitchell killed in } \\
\text { military service, } \\
\text { aeroplane crash over } \\
\text { English Channel } \\
\text { (15 December) }\end{array}$ & $\begin{array}{l}\text { Georgia O'Keeffe, } \\
\text { Pelvis IV } \\
\text { Horace Pippin, } \\
\text { Interior } \\
\text { Johnny Everyman } \\
\text { (educational comics } \\
\text { series) launches a four- } \\
\text { year run }\end{array}$ \\
\hline
\end{tabular}




\begin{tabular}{|c|c|c|c|c|}
\hline Date & Events & Criticism & Literature & Performance \\
\hline 1945 & 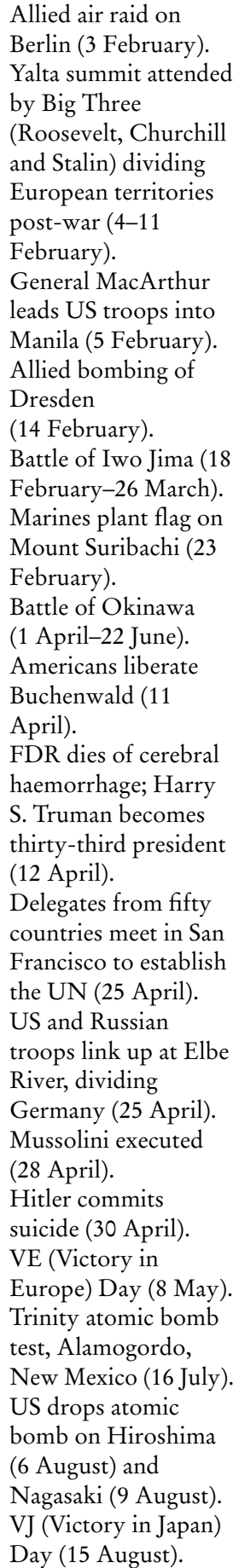 & $\begin{array}{l}\text { Norman Cousins, } \\
\text { Modern Man is } \\
\text { Obsolete } \\
\text { Hannah Arendt, } \\
\text { 'German Guilt' } \\
\text { St Clair Drake and } \\
\text { Horace R. Cayton, } \\
\text { Black Metropolis: A } \\
\text { Study of Negro Life } \\
\text { in a Northern City } \\
\text { Walter White, } \\
\text { A Rising Wind } \\
\text { W. E. B. Du Bois, } \\
\text { Color and } \\
\text { Democracy }\end{array}$ & $\begin{array}{l}\text { John Hersey wins } \\
\text { Pulitzer Prize for A } \\
\text { Bell for Adano } \\
\text { Chester Himes, If } \\
\text { He Hollers, Let Him } \\
\text { Go } \\
\text { John Steinbeck, } \\
\text { Cannery Row } \\
\text { E. B. White, Stuart } \\
\text { Little (children's } \\
\text { story) } \\
\text { Richard Wright, } \\
\text { Black Boy }\end{array}$ & $\begin{array}{l}\text { Tennessee Williams, } \\
\text { The Glass Menagerie } \\
\text { (drama) } \\
\text { Richard Rodgers } \\
\text { and Oscar } \\
\text { Hammerstein II, } \\
\text { Carousel (musical) } \\
\text { Sigmund Romberg, } \\
\text { Up in Central Park } \\
\text { (musical) } \\
\text { Arthur Laurents, } \\
\text { Home of the Brave } \\
\text { (drama) } \\
\text { Mary Chase, } \\
\text { Harvey (comedy) }\end{array}$ \\
\hline
\end{tabular}




\begin{tabular}{|c|c|c|c|}
\hline Film & Radio & Music & Art \\
\hline $\begin{array}{l}1944 \text { Oscars awarded - } \\
\text { Going My Way wins } \\
\text { Best Picture, Best } \\
\text { Director (Leo } \\
\text { McCarey), and Best } \\
\text { Actor (Bing Crosby) } \\
\text { Mildred Pierce } \\
\text { (Michael Curtiz) } \\
\text { The Lost Weekend } \\
\text { (Billy Wilder) } \\
\text { Spellbound (Alfred } \\
\text { Hitchcock) } \\
\text { They Were Expendable } \\
\text { (John Ford) } \\
\text { The Bells of St. Mary's } \\
\text { (Leo McCarey) }\end{array}$ & $\begin{array}{l}\text { Beulab (1945-54) } \\
\text { Queen for a Day } \\
\text { (1945-57) } \\
\text { Lionel Barrymore } \\
\text { replaces Cecil B. } \\
\text { DeMille as host of Lux } \\
\text { Radio Theatre } \\
\text { Academy Awards are } \\
\text { radio broadcast in their } \\
\text { entirety for the first } \\
\text { time }\end{array}$ & $\begin{array}{l}\text { Bing Crosby, Merry } \\
\text { Christmas (album) } \\
\text { Nat King Cole, Nat } \\
\text { King Cole Trio (album) } \\
\text { Dizzy Gillespie, 'Salt } \\
\text { Peanuts' } \\
\text { Bill Monroe, 'Blue } \\
\text { Moon of Kentucky' } \\
\text { Fred Kirby, 'Atomic } \\
\text { Power' }\end{array}$ & $\begin{array}{l}\text { Willem de Kooning, } \\
\text { Study for Pink Angels } \\
\text { and Still Life } \\
\text { Jackson Pollock and } \\
\text { Lee Krasner purchase } \\
\text { home and studio in } \\
\text { East Hampton, New } \\
\text { York }\end{array}$ \\
\hline
\end{tabular}




\begin{tabular}{|c|c|c|c|c|}
\hline Date & Events & Criticism & Literature & Performance \\
\hline 1946 & $\begin{array}{l}\text { Truman establishes } \\
\text { the Central } \\
\text { Intelligence Agency } \\
\text { (CIA). } \\
\text { First issue of } \\
\text { Roosevelt dime } \\
\text { (10-cent piece), on } \\
\text { Roosevelt's birthday } \\
\text { (30 January). } \\
\text { ENIAC (first large- } \\
\text { scale computer) } \\
\text { performs first } \\
\text { function. } \\
\text { George Kennan sends } \\
\text { 'long telegram' from } \\
\text { Moscow to US State } \\
\text { Department (22 } \\
\text { February). } \\
\text { Winston Churchill's } \\
\text { 'Iron Curtain' speech } \\
\text { at Westminster } \\
\text { College, Fulton, } \\
\text { Missouri ( } 5 \text { March). } \\
\text { 'Operation } \\
\text { Crossroads' atomic } \\
\text { bomb test at Bikini } \\
\text { Atoll, Marshall } \\
\text { Islands (1 July). } \\
\text { Bikini bathing suit } \\
\text { introduced. }\end{array}$ & $\begin{array}{l}\text { John Hersey, } \\
\text { Hiroshima } \\
\text { Benjamin Spock, } \\
\text { The Common Book } \\
\text { of Baby and Child } \\
\text { Care } \\
\text { Malcolm Cowley, } \\
\text { The Portable } \\
\text { Faulkner } \\
\text { Clement Greenberg, } \\
\text { 'Avant Garde and } \\
\text { Kitsch' }\end{array}$ & $\begin{array}{l}\text { William Carlos } \\
\text { Williams, Patterson } \\
\text { (long poem) } \\
\text { Robert Penn } \\
\text { Warren, All the } \\
\text { King's Men } \\
\text { Eudora Welty, Delta } \\
\text { Wedding }\end{array}$ & $\begin{array}{l}\text { Garson Kanin, Born } \\
\text { Yesterday (comedy) } \\
\text { Irving Berlin, Annie } \\
\text { Get Your Gun } \\
\text { (musical) } \\
\text { Duke Ellington and } \\
\text { John LaTouche, } \\
\text { Beggar's Holiday } \\
\text { (musical) } \\
\text { Cole Porter and } \\
\text { Orson Welles, } \\
\text { Around the World } \\
\text { (musical) } \\
\text { Eugene O'Neill, The } \\
\text { Iceman Cometh } \\
\text { (drama) } \\
\text { Harold Arlen and } \\
\text { Johnny Mercer, St. } \\
\text { Louis Woman } \\
\text { (musical) } \\
\text { First Tony Awards }\end{array}$ \\
\hline
\end{tabular}




\begin{tabular}{|c|c|c|c|}
\hline Film & Radio & Music & Art \\
\hline $\begin{array}{l}1945 \text { Oscars awarded - } \\
\text { The Lost Weekend } \\
\text { wins Best Picture, Best } \\
\text { Director (Billy Wilder) } \\
\text { and Best Actor (Ray } \\
\text { Milland) } \\
\text { It's a Wonderful Life } \\
\text { (Frank Capra) } \\
\text { The Big Sleep (Howard } \\
\text { Hawks) } \\
\text { The Best Years of Our } \\
\text { Lives (William Wyler) } \\
\text { Duel in the Sun (King } \\
\text { Vidor) } \\
\text { The Killers (Robert } \\
\text { Siodmak) } \\
\text { Notorious (Alfred } \\
\text { Hitchcock) } \\
\text { My Darling } \\
\text { Clementine (John } \\
\text { Ford) }\end{array}$ & $\begin{array}{l}\text { Twenty Questions } \\
\text { (1946-54) } \\
\text { Artbur Godfrey's } \\
\text { Talent Scouts (1946-56) } \\
\text { The Bickersons } \\
(1946-51)\end{array}$ & $\begin{array}{l}\text { Louis Jordan and his } \\
\text { Tympani Five, 'Choo } \\
\text { Choo Ch' Boogie' } \\
\text { Nat King Cole Trio, } \\
\text { 'The Christmas Song' } \\
\text { Duke Ellington, 'Take } \\
\text { the A Train' } \\
\text { Stan Kenton, 'Shoo Fly } \\
\text { Pie and Apple Pan } \\
\text { Dowdy' (vocals by } \\
\text { June Christy) } \\
\text { Charlie 'Yardbird' } \\
\text { Parker and Dizzy } \\
\text { Gillespie play Carnegie } \\
\text { Hall }\end{array}$ & $\begin{array}{l}\text { Jacob Lawrence, } \\
\text { Seamstress } \\
\text { Architect Mies van der } \\
\text { Rohe begins } \\
\text { Farnsworth House } \\
\text { (1946-51) } \\
\text { Art critic Robert } \\
\text { Coates coins the term } \\
\text { 'Abstract } \\
\text { Expressionism' } \\
\text { Peggy Guggenheim } \\
\text { publishes memoirs, } \\
\text { Out of this Century } \\
\text { Romare Bearden } \\
\text { criticises Harmon } \\
\text { Foundation for } \\
\text { supporting 'mediocre' } \\
\text { art }\end{array}$ \\
\hline
\end{tabular}




\begin{tabular}{|c|c|c|c|c|}
\hline Date & Events & Criticism & Literature & Performance \\
\hline 1947 & $\begin{array}{l}\text { Arabs and Jews reject } \\
\text { a British offer to split } \\
\text { Palestine. } \\
\text { Truman promulgates } \\
\text { Truman Doctrine, to } \\
\text { halt the growth of } \\
\text { communism in } \\
\text { Europe (12 March). } \\
\text { Truman issues } \\
\text { Executive Order } \\
\text { 9835, requiring } \\
\text { federal employees to } \\
\text { swear a loyalty oath. } \\
\text { At the Big Four } \\
\text { meeting in Moscow, } \\
\text { Secretary of State } \\
\text { George C. Marshall } \\
\text { inaugurates the } \\
\text { Marshall Plan, to aid } \\
\text { reconstruction of } \\
\text { Europe with western } \\
\text { support. } \\
\text { Jackie Robinson } \\
\text { signed to play for } \\
\text { Brooklyn Dodgers, } \\
\text { first African } \\
\text { American to play for } \\
\text { a major-league } \\
\text { baseball team (12 } \\
\text { April); named } \\
\text { 'Rookie of the Year' } \\
\text { (19 September). } \\
\text { Jewish refugees } \\
\text { aboard the Exodus } \\
\text { turned back by } \\
\text { British (18 July). } \\
\text { After labour strikes } \\
\text { throughout the } \\
\text { preceding two years, } \\
\text { Senate approves } \\
\text { Taft-Hartley Act, } \\
\text { limiting the power of } \\
\text { unions. }\end{array}$ & $\begin{array}{l}\text { Henry L. Stimson, } \\
\text { 'The Decision to } \\
\text { Use the Atom } \\
\text { Bomb' } \\
\text { Walter Lippmann, } \\
\text { The Cold War: A } \\
\text { Study in US Foreign } \\
\text { Policy } \\
\text { Erich Fromm, Man } \\
\text { for Himself: An } \\
\text { Inquiry into the } \\
\text { Psychology of Ethics } \\
\text { Ferdinand Lundberg } \\
\text { and Marynia F. } \\
\text { Farnham, Modern } \\
\text { Woman: The Lost } \\
\text { Sex } \\
\text { Anne Frank, The } \\
\text { Diary of Anne Frank } \\
\text { Cleanth Brooks, The } \\
\text { Well Wrought Urn }\end{array}$ & $\begin{array}{l}\text { James A. Michener, } \\
\text { Tales of the South } \\
\text { Pacific } \\
\text { Margaret Wise } \\
\text { Brown, Goodnight } \\
\text { Moon (children's } \\
\text { story) } \\
\text { Truman Capote's } \\
\text { Others Voices, Other } \\
\text { Rooms } \\
\text { Mickey Spillane, } \\
\text { I, the Jury }\end{array}$ & $\begin{array}{l}\text { Arthur Miller, All } \\
\text { My Sons } \\
\text { Tennessee Williams, } \\
\text { A Streetcar Named } \\
\text { Desire } \\
\text { Alan Jay Lerner and } \\
\text { Frederick Lowe, } \\
\text { Brigadoon (musical) } \\
\text { Burton Lane and } \\
\text { E. Y. Harburg, } \\
\text { Finian's Rainbow } \\
\text { (musical) } \\
\text { Kurt Weill, } \\
\text { Langston Hughes, } \\
\text { and Elmer Rice, } \\
\text { Street Scene } \\
\text { (musical) }\end{array}$ \\
\hline
\end{tabular}




\begin{tabular}{|c|c|c|c|}
\hline Film & Radio & Music & Art \\
\hline $\begin{array}{l}1946 \text { Oscars awarded - } \\
\text { The Best Years of Our } \\
\text { Lives wins Best } \\
\text { Picture, Best Director } \\
\text { (William Wyler), Best } \\
\text { Actor (Fredric March), } \\
\text { and Best Supporting } \\
\text { Actor (Harold Russell) } \\
\text { House Un-American } \\
\text { Activities Committee } \\
\text { convenes to investigate } \\
\text { communist activity in } \\
\text { Hollywood } \\
\text { Crossfire (Edward } \\
\text { Dmytryk) } \\
\text { A Double Life (George } \\
\text { Cukor) } \\
\text { Gentleman's } \\
\text { Agreement (Elia } \\
\text { Kazan) }\end{array}$ & $\begin{array}{l}\text { You Bet Your Life } \\
\text { (1947-50) } \\
\text { My Friend Irma } \\
\text { (1947-54) } \\
\text { Margaret Truman } \\
\text { (daughter of Harry } \\
\text { Truman) has vocal } \\
\text { debut for national } \\
\text { radio audience }\end{array}$ & $\begin{array}{l}\text { Frankie Laine, 'All of } \\
\text { Me' } \\
\text { Dick Haymes, 'How } \\
\text { are Things in Glocca } \\
\text { Morra?' } \\
\text { The Weavers (folk } \\
\text { group) begin } \\
\text { performing }\end{array}$ & $\begin{array}{l}\text { Jackson Pollock } \\
\text { produces his first 'drip } \\
\text { paintings', paints } \\
\text { Cathedral } \\
\text { Norman Rockwell } \\
\text { begins Four Seasons } \\
\text { calendars for Brown } \\
\text { and Bigelow }\end{array}$ \\
\hline
\end{tabular}




\begin{tabular}{|c|c|c|c|c|}
\hline Date & Events & Criticism & Literature & Performance \\
\hline 1948 & $\begin{array}{l}\text { Berlin Airlift begins, } \\
\text { bringing food and } \\
\text { supplies by air into } \\
\text { blockaded West } \\
\text { Berlin, sustaining } \\
\text { residents until } \\
\text { blockades were lifted. } \\
\text { (26 June-12 May } \\
\text { 1949). } \\
\text { Truman signs } \\
\text { Executive Order } \\
\text { 9981, desegregating } \\
\text { armed forces (26 } \\
\text { July). } \\
\text { Whitaker Chambers } \\
\text { admits to 1930s-era } \\
\text { communist espionage } \\
\text { activity to HUAC, } \\
\text { implicates Algier } \\
\text { Hiss. } \\
\text { After intense conflict } \\
\text { in Palestine, leading } \\
\text { to entrenchment of } \\
\text { Israeli state, flag of } \\
\text { Israel adopted. } \\
\text { In election-night } \\
\text { upset, Truman wins } \\
\text { second term; 'Dewey } \\
\text { Defeats Truman' } \\
\text { incorrectly printed in } \\
\text { the next morning's } \\
\text { Chicago Daily } \\
\text { Tribune (2 } \\
\text { November). }\end{array}$ & $\begin{array}{l}\text { Alfred Kinsey's } \\
\text { Sexual Behavior in } \\
\text { the Human Male } \\
\text { Francis E Merrill, } \\
\text { Social Problems and } \\
\text { the Homefront: A } \\
\text { Study of War-time } \\
\text { Influences }\end{array}$ & $\begin{array}{l}\text { T. S. Eliot wins } \\
\text { Nobel Prize in } \\
\text { Literature } \\
\text { Irwin Shaw, The } \\
\text { Young Lions } \\
\text { William Faulkner, } \\
\text { Intruder in the Dust } \\
\text { Zora Neale } \\
\text { Hurston, Seraph on } \\
\text { the Sewanee } \\
\text { Norman Mailer, The } \\
\text { Naked and the Dead } \\
\text { Shirley Jackson, } \\
\text { 'The Lottery' (short } \\
\text { story) }\end{array}$ & $\begin{array}{l}\text { Second Annual } \\
\text { Tony Awards - Best } \\
\text { Play: Mr. Roberts } \\
\text { (beating A Streetcar } \\
\text { Named Desire) } \\
\text { Milton Berle debuts } \\
\text { as host of NBC TV's } \\
\text { Texaco Star Theater } \\
\text { Tennessee Williams, } \\
\text { Summer and Smoke } \\
\text { (drama) } \\
\text { Frank Loesser, } \\
\text { Where's Charley? } \\
\text { (musical comedy) } \\
\text { Cole Porter, Kiss } \\
\text { Me, Kate (musical) } \\
\text { Jule Styne and } \\
\text { Sammy Cahn, High } \\
\text { Button Shoes }\end{array}$ \\
\hline
\end{tabular}




\begin{tabular}{llll}
\hline Film & Radio & Music & Art \\
\hline $\begin{array}{l}\text { 1947 Oscars awarded - } \\
\text { Gentleman's }\end{array}$ & $\begin{array}{l}\text { Life with Luigi } \\
(1948-52)\end{array}$ & $\begin{array}{l}\text { Dinah Shore, 'Buttons } \\
\text { and Bows' }\end{array}$ & $\begin{array}{l}\text { Walt Kelly, Pogo } \\
\text { (comic strip) }\end{array}$ \\
$\begin{array}{l}\text { Agreement } \text { wins Best } \\
\text { Our Miss Brooks } \\
\text { Director (Elia Kazan) }\end{array}$ & $\begin{array}{l}\text { Vaugh Monroe and } \\
\text { the Sons of the }\end{array}$ & $\begin{array}{l}\text { Andrew Wyeth, } \\
\text { Christina's World }\end{array}$ \\
$\begin{array}{l}\text { Force of Evil (Abraham } \\
\text { Polonsky) }\end{array}$ & $\begin{array}{l}\text { Pioneers, 'Cool Water' } \\
\text { (western) }\end{array}$ & $\begin{array}{l}\text { Jackson Pollock, No. 5, } \\
\text { 1948 }\end{array}$ \\
Johnny Belinda (Jean & Moses Asch founds & Anne Redpath, \\
Negulesco) & Folkways Records & Window in Menton \\
The Lady from & John Cage, Suite for & After a year filled with \\
Shanghai (Orson & Toy Piano & tragedy, Arshile Gorky \\
Welles) & Aaron Copeland, Red & commits suicide \\
Red River (Howard & Pony Suite & \\
Hawks) & & \\
The Snake Pit and & & \\
Sorry, Wrong Number & & & \\
(Anatole Litvak) & & & \\
& & &
\end{tabular}




\begin{tabular}{|c|c|c|c|c|}
\hline Date & Events & Criticism & Literature & Performance \\
\hline 1949 & $\begin{array}{l}\text { North Atlantic Treaty } \\
\text { enacted ( } 24 \text { August). } \\
\text { Riot prevents Paul } \\
\text { Robeson from singing } \\
\text { in Peekskill, New } \\
\text { York (28 August). } \\
\text { Soviet Union } \\
\text { detonates its first } \\
\text { atomic bomb } \\
\text { (28 August). } \\
\text { Television sales hit } \\
60,000 \text { per week. } \\
\text { First Emmy Awards - } \\
\text { Most Outstanding } \\
\text { Television } \\
\text { Personality: Shirley } \\
\text { Dinsdale Layburn } \\
\text { and her puppet Judy } \\
\text { Splinters. } \\
\text { Look magazine } \\
\text { proclaims radio } \\
\text { 'doomed' to takeover } \\
\text { by television within } \\
\text { three years. } \\
\text { Board games: } \\
\text { Candvland and Clue. }\end{array}$ & $\begin{array}{l}\text { Margaret Mead, } \\
\text { Male and Female } \\
\text { Joseph Campbell, } \\
\text { The Hero With a } \\
\text { Thousand Faces }\end{array}$ & $\begin{array}{l}\text { William Faulkner } \\
\text { wins Nobel Prize } \\
\text { for literature } \\
\text { Nelson Algren, The } \\
\text { Man with the } \\
\text { Golden Arm } \\
\text { Paul Bowles, The } \\
\text { Sheltering Sky }\end{array}$ & $\begin{array}{l}\text { Third Annual Tony } \\
\text { Awards - Best Play: } \\
\text { Death of a Salesman; } \\
\text { Best Musical: Kiss } \\
\text { Me, Kate } \\
\text { Arthur Miller wins } \\
\text { Pulitzer Prize for } \\
\text { drama } \\
\text { Kurt Weill and } \\
\text { Maxwell Anderson, } \\
\text { Lost in the Stars } \\
\text { (musical drama) } \\
\text { Arthur Miller, Death } \\
\text { of a Salesman } \\
\text { T. S. Eliot, The } \\
\text { Cocktail Party } \\
\text { Richard Rodgers } \\
\text { and Oscar } \\
\text { Hammerstein II, } \\
\text { South Pacific } \\
\text { (musical) } \\
\text { Jule Styne and Leo } \\
\text { Robin, Gentlemen } \\
\text { Prefer Blondes } \\
\text { (comedy) }\end{array}$ \\
\hline
\end{tabular}




\begin{tabular}{|c|c|c|c|}
\hline Film & Radio & Music & Art \\
\hline $\begin{array}{l}1948 \text { Oscars awarded - } \\
\text { Hamlet wins Best } \\
\text { Picture and Best Actor } \\
\text { (Laurence Olivier) } \\
\text { All the King's Men } \\
\text { (Robert Rossen) } \\
\text { Pinky (Elia Kazan and } \\
\text { John Ford) } \\
\text { Intruder in the Dust } \\
\text { (Clarence Brown) } \\
\text { The Heiress (William } \\
\text { Wyler) } \\
\text { She Wore a Yellow } \\
\text { Ribbon (John Ford) } \\
\text { The Third Man (Carol } \\
\text { Reed) } \\
\text { White Heat (Raoul } \\
\text { Walsh) } \\
\text { Adam's Rib (George } \\
\text { Cukor) } \\
\text { Cinderella (Walt } \\
\text { Disney) }\end{array}$ & $\begin{array}{l}\text { Yours Truly, Johnny } \\
\text { Dollar (1949-62) } \\
\text { Crime Does Not Pay } \\
\text { (1949-52) } \\
\text { Dr. Kildare (1949-51) } \\
\text { Father Knows Best } \\
\text { (1949-53) }\end{array}$ & $\begin{array}{l}\text { Ray Charles's first } \\
\text { recording, 'Confession } \\
\text { Blues' } \\
\text { Birdland (jazz club) } \\
\text { opens in New York } \\
\text { Anthony Benedetto } \\
\text { changes stage name to } \\
\text { Tony Bennett at the } \\
\text { suggestion of Bob } \\
\text { Hope } \\
\text { 'You're Breaking my } \\
\text { Heart' recorded by } \\
\text { Buddy Clark (who dies } \\
\text { in an aeroplane crash, } \\
1 \text { October), Vic } \\
\text { Damone, and The } \\
\text { Inkspots } \\
\text { Hank Williams, } \\
\text { 'Lovesick Blues' }\end{array}$ & $\begin{array}{l}\text { Willem de Kooning, } \\
\text { Woman } \\
\text { Barnett Newman, } \\
\text { Abraham } \\
\text { Robert Motherwell, } \\
\text { Five in the Afternoon } \\
\text { Philip Johnson, Glass } \\
\text { House (architecture) }\end{array}$ \\
\hline
\end{tabular}


